\title{
Detecting mid-infrared light by molecular frequency upconversion with dual-wavelength hybrid nanoantennas
}

Angelos Xomalis ${ }^{1}$, Xuezhi Zheng ${ }^{1,2}$, Rohit Chikkaraddy ${ }^{1}$, Zsuzsanna Koczor-Benda ${ }^{3}$, Ermanno Miele ${ }^{1,4,5}$, Edina Rosta ${ }^{3}$, Guy A E Vandenbosch ${ }^{2}$, Alejandro Martínez ${ }^{6}$, Jeremy J Baumberg ${ }^{1^{*}}$

${ }^{1}$ NanoPhotonics Centre, Cavendish Laboratory, Department of Physics, University of Cambridge, Cambridge, United Kingdom.

${ }^{2}$ Department of Electrical Engineering (ESAT-TELEMIC), KU Leuven, Leuven, Belgium

${ }^{3}$ Department of Physics and Astronomy, University College London, London, United Kingdom

${ }^{4}$ Department of Chemistry, University of Cambridge, Cambridge, United Kingdom

${ }^{5}$ The Faraday Institution, Harwell Science and Innovation Campus, Oxford, United Kingdom

${ }^{6}$ Nanophotonics Technology Center, Universitat Politècnica de València, Valencia, Spain

*Corresponding author. email: jjb12@cam.ac.uk

\section{ABSTRACT}

Coherent interconversion of signals between optical and mechanical domains is enabled by optomechanical interactions. Extreme light-matter coupling produced by confining light to nanoscale mode volumes can then access single mid-infrared (MIR) photon sensitivity. Here we utilise the infrared absorption and Raman activity of molecular vibrations in plasmonic nanocavities to demonstrate frequency upconversion. We convert $\lambda \sim 10 \mu \mathrm{m}$ incoming light to visible via surface-enhanced Raman scattering (SERS) in doubly-resonant antennas that enhance upconversion by $>10^{10}$. We show $>200 \%$ amplification of the SERS antiStokes emission when a MIR pump is tuned to a molecular vibrational frequency, obtaining lowest detectable powers $\sim 1 \mu \mathrm{W} / \mu \mathrm{m}^{2}$ at room temperature. These results have potential for lowcost and large-scale infrared detectors and spectroscopic techniques, and bring singlemolecule sensing into the infrared.

One-Sentence Summary:

Molecules as Mixers.

Detecting weak mid-infrared light currently requires expensive cooled devices. Xomalis et $a l$ instead convert the light to visible frequencies where it is efficiently measured, by using vibrating molecules inside a cavity that traps the multiple frequencies of light simultaneously. Using self-assembled devices integrated onto silicon wafers, they exploit the nonlinear interaction of mechanical vibrations and oscillations of light. This yields the first successful devices in the recently emerging field of molecular optomechanics.

Infrared spectroscopy delivers information hard to obtain from other frequency bands, such as atmospheric absorption of molecules (greenhouse gases) or thermally-emitted radiation 
from earth (meteorological maps or imaging wildfires) (1-5). While development of mid-IR (MIR) sources evolves, a bottleneck continues to be in producing low-noise roomtemperature detectors (6). One proposed scheme is to directly upconvert MIR photons into high-energy visible photons that are efficiently detected, potentially delivering single-photon semiconductor-based detectors (7-9). Analogous wavelength conversion from microwave to optical frequencies has utilised expensive fabrication and cryogenic temperatures $(10,11)$, as well as $\mathrm{LiNbO}_{3}$ resonators $(12,13)$. Critically, in order to access the efficiencies required, strongly-enhanced light-matter interactions are paramount. Thus, plasmonic devices and planar resonant metasurfaces which confine light have been of interest for MIR integrated detection and biosensing (14-16).

A promising approach for detecting infrared radiation through frequency upconversion is via molecular optomechanical coupling (17). Optomechanical interactions allow coherent conversion of signals between optical and mechanical domains (Fig. 1). Nanocavities containing vibrating molecules act as mechanical oscillators, with MIR-absorbing infrared vibrational modes probed by a visible laser through their Raman scattering (Fig. 1B). The required interactions can be boosted by using the tight light localisation inside plasmonic nanocavities $<100 \mathrm{~nm}$ across which yield detectable signals even from single vibrational bonds (18). The interaction of light and matter in these sub-nm mode volumes gives extreme optomechanical coupling with single MIR-photon sensitivity in principle, but so far only studied theoretically (17). The noise-equivalent power of hybrid nanocavity-molecular detectors is predicted to be hundred-fold lower than commercial uncooled detectors.
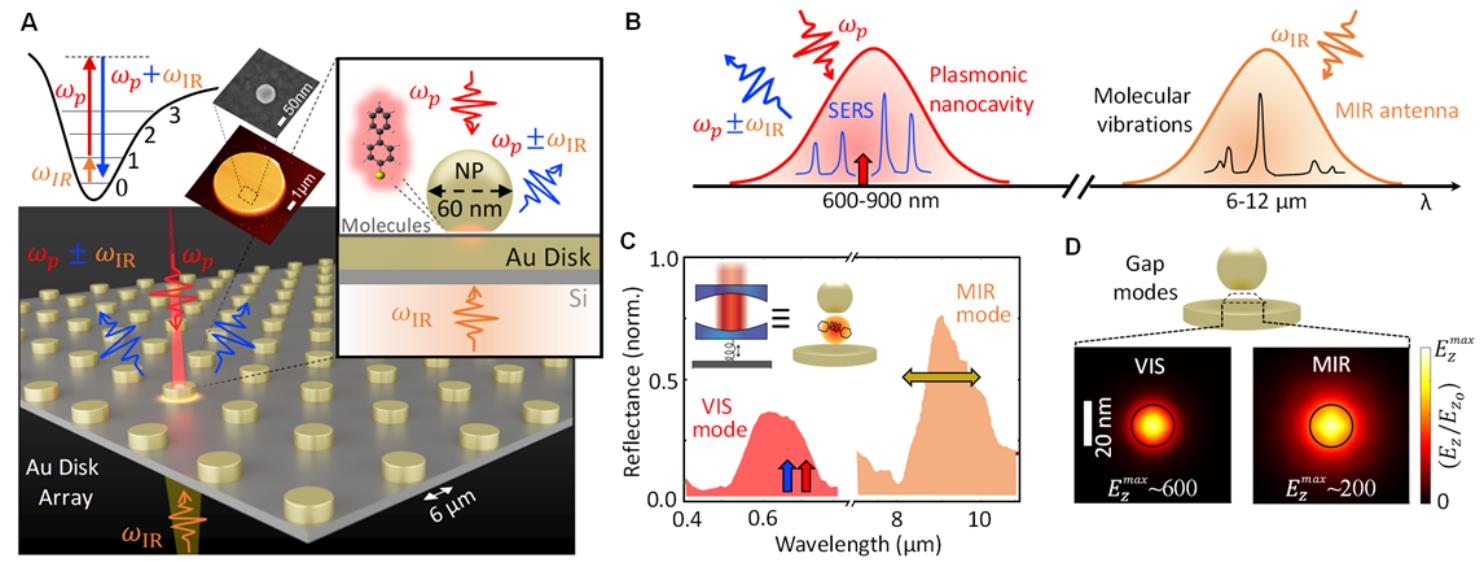

Fig. 1. Dual-wavelength antenna and frequency upconversion. (A) Pump (MIR) - probe (visible) detection configuration. Inset shows upconversion process, AFM (disk) and SEM (nanoparticle) images, and self-assembled monolayer of biphenyl-4-thiol which creates $1.3 \mathrm{~nm}$ cavity between $60 \mathrm{~nm}$ $\mathrm{Au}$ nanoparticle and $6 \mu \mathrm{m}$ disk. (B) Scheme of MIR to visible up-conversion via molecular optomechanics. (C) Experimental reflectance of NPoR resonances at both visible (red) and MIR (orange) wavelengths. Arrows indicate SERS probe wavelength $(785 \mathrm{~nm}$, red), inelastic scattered light (blue) and MIR tuning range (8.5-12.6 $\mu \mathrm{m}$, yellow). Inset shows equivalence of optomechanical cavity and NPoR. (D) Near-field normalised maps of mid-infrared (MIR) and visible gap modes of NPoR. Black circle shows $20 \mathrm{~nm}$ nanoparticle facet. 
Of vital importance for upconversion efficiency is the optimal spatial overlap of visible and infrared radiation. Plasmonics allows extreme light confinement at visible frequencies, but at longer wavelengths light localisation drops significantly. Achieving light confinement simultaneously in both visible and MIR spectral regions requires a hybrid dual resonator (19). Here, this is fulfilled by creating doubly-resonant antennas which focus long- and shortwavelengths into the same active region allowing good optomechanical coupling (Fig. 1D). Their construction combines bottom-up and top-down methods that allow for ease of fabrication and cost-effective large-scale arrays of devices.

To demonstrate MIR detection, we perform surface-enhanced Raman spectroscopy (SERS) on self-assembled molecular monolayers (SAMs) with discrete vibrational absorption modes in the $\lambda=6-12 \mu \mathrm{m}$ range. Coupling requires matching the optical (infrared absorption) and mechanical (molecular vibration) energies. Biphenyl-4-thiol (BPT) is chosen (inset Fig. 1A) since it provides vibrations that are simultaneously active in both IR absorption and Raman, and binds strongly and consistently to gold. Integrated into a dual-wavelength gold antenna, the nanoparticle-on-resonator (NPOR), this strongly confines visible and MIR within the same active region (19), accessing single-molecule optomechanical nonlinearities $(20,21)$. The $A u$ disk resonators (diameter $6 \mu \mathrm{m}$ ) have a fundamental resonant mode around $\lambda=10 \mu \mathrm{m}$ and high order modes in the visible (19). Onto these is self-assembled a molecular monolayer of BPT, with $60 \mathrm{~nm}$ Au nanoparticles drop-cast on top. The molecule length sets the $1.3 \mathrm{~nm}$ spacing (18), giving resonances which are experimentally measured with visible and MIR light (Fig. 1C). Comparing with simulations shows field enhancements $E / E_{0}>500$ (visible) and $>200$ (MIR) (Fig. S4A) (19), providing a more favourable geometry than previously devised for (simulating) molecular upconversion (17). A modified microscope focusses visible and MIR lasers onto the same NPoR device (with $>40$ NPoRs tested here). The $1080 \mathrm{~cm}^{-1}$ molecular vibration is observed in SERS antiStokes emission, with amplitude that increases linearly when pumped directly with MIR radiation tuned to the same energy.

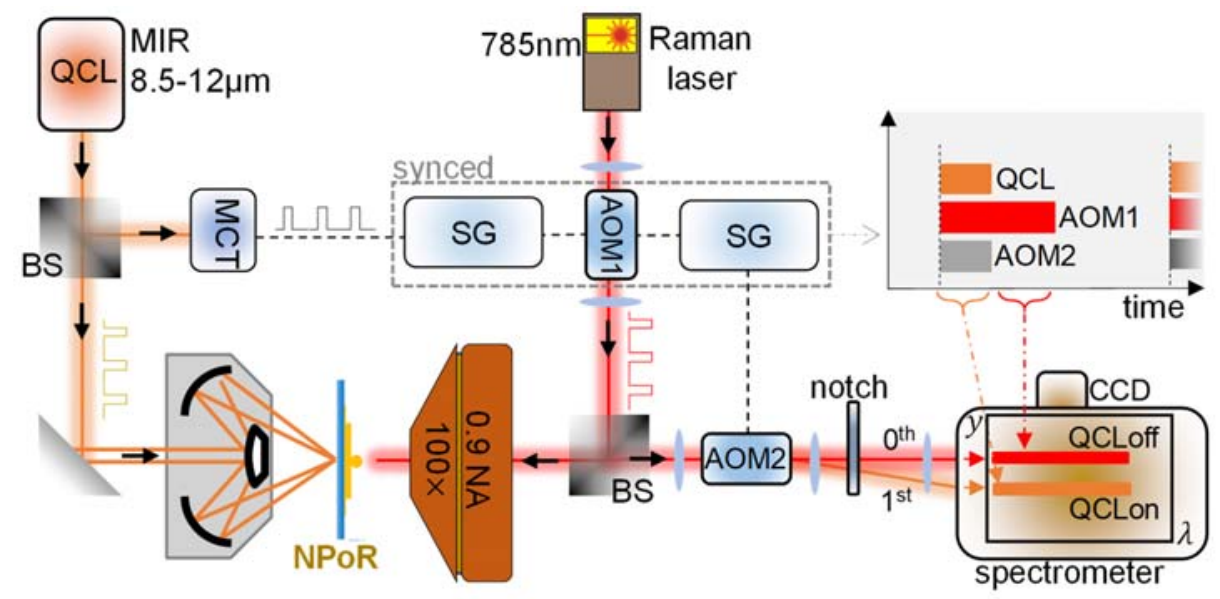

Fig. 2. MIR and visible spectroscopy. Dual microscope combines visible probe and MIR pump for frequency upconversion of molecules in nanogaps: AOM (acousto-optical modulator), MCT (mercurycadmium-telluride detector), BS (beamsplitter), SG (signal generator). Inset: Timing sequence of each repetition of QCL (pump) to Raman laser modulation (AOM1), where AOM2 deflects SERS spectrum to different vertical position $y$ on spectrometer slit. 
A

C
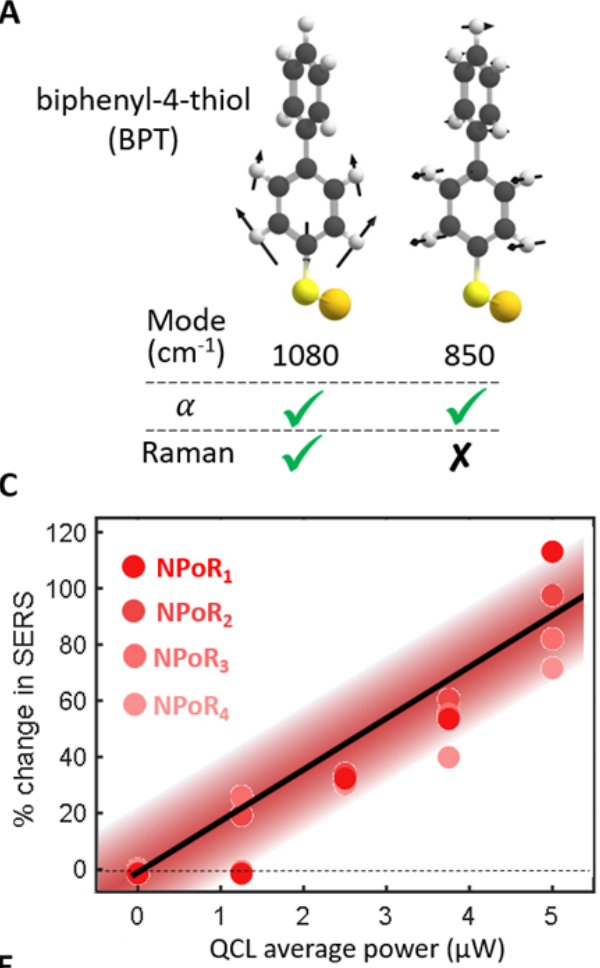

$\mathbf{E}$

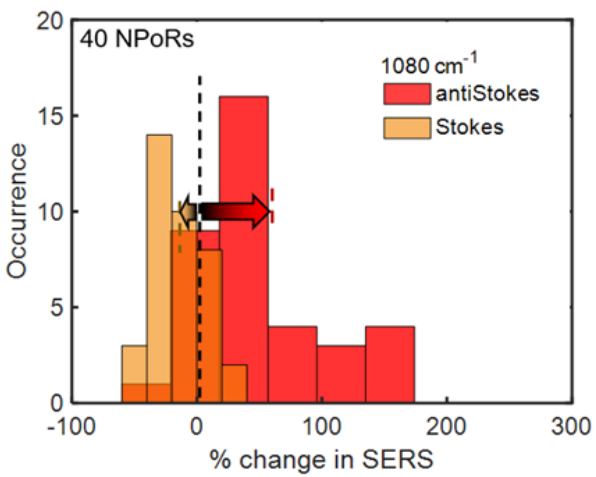

B
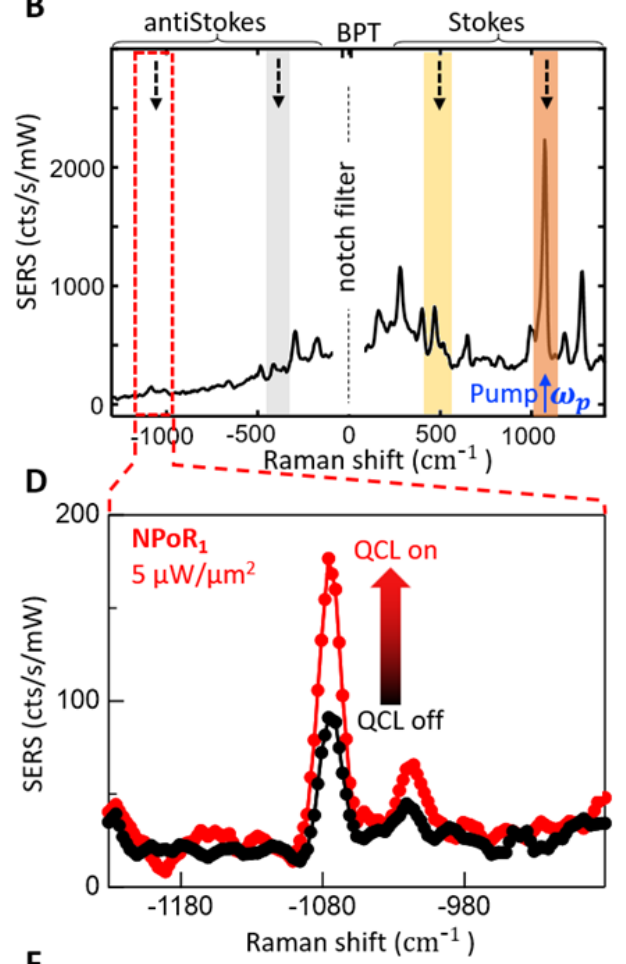

$\mathbf{F}$

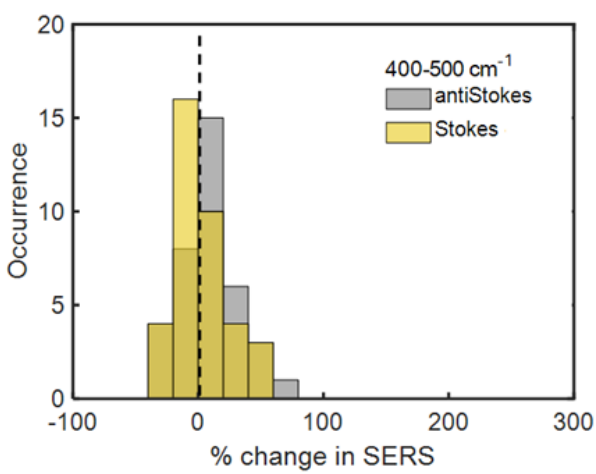

Fig. 3. Upconversion of MIR to visible photons in hybrid plasmonic antennas. (A) Vibrations of BPT, showing frequencies with strong infrared absorption $(\alpha)$ or Raman. (B) BPT SERS spectrum from $785 \mathrm{~nm}$ probe alone. Shaded regions mark pump (orange, $1080 \mathrm{~cm}^{-1}$ ) and monitored frequency bands (arrows). (C) Power dependence for 4 NPoRs. (D) Raw spectra showing $v=1080 \mathrm{~cm}^{-1}$ antiStokes increase when MIR pump is on (red). (E-F) MIR-induced change in SERS of 40 NPoRs at Stokes and antiStokes peaks at (E) $1080 \mathrm{~cm}^{-1}$ and (F) $400-500 \mathrm{~cm}^{-1}$.

Our experiments use synchronised visible and quantum cascade laser ( $Q C L$ ) rectangular pulses $(0.4 \mu \mathrm{s})$ to collect SERS spectra with/out the MIR light (Fig. 2). These confirm the prediction of upconversion (17), using the $v=1080 \mathrm{~cm}^{-1}$ BPT mode which is both infrared and Raman active (Fig. 3A, 4A). Measuring SERS from NPoRs shows the expected BPT vibrations on both Stokes and antiStokes sides of the laser (Fig. 3B), which are stable and repeatable over long periods. The $\mathrm{QCL}$ is then tuned to the same photon energy $h v$ (orange, Fig. $3 \mathrm{~B}$ ) and an infrared pump power dependence recorded (Fig. 3C). We find that the antiStokes SERS is $200 \%$ higher when QCL average powers of $5 \mu \mathrm{W} / \mu \mathrm{m}^{2}$ are incident (Fig. 3D, using peak area 
ratio $\mathrm{AS}\left(\mathrm{QCL}_{\mathrm{on}}\right) / \mathrm{AS}\left(\mathrm{QCL}_{\mathrm{off}}\right)$ with background-subtracted antiStokes peaks, see Methods, S2). The expected linear dependence of frequency upconversion with pump power is similar for different NPoRs (red points, Fig. 3C). The lowest detectable light intensity of these dualwavelength plasmonic antennas is $\sim 1 \mu \mathrm{W} / \mu \mathrm{m}^{2}$ (Fig. 3C), while the lock-in detection synchronised technique here shows that the response speed is sub- $\mu \mathrm{s}$, much faster than the $\mathrm{QCL}$ pulse repetition rate $(5 \mu \mathrm{s})$.

To better quantify the upconversion efficiency, we measure the percentage change of SERS on 40 NPoRs, where each NP is located at different positions on its disk antenna. These show an average $52 \%$ increase of antiStokes at $v$ (red, Fig. 3E) for $5 \mu \mathrm{W} / \mu \mathrm{m}^{2} \mathrm{MIR}$, while by contrast the Stokes at $v$ shows a decrease of $13 \%$ (red). No systematic correlation with the NP position on the disk is apparent, though likely it controls in-coupling of both visible and MIR light into the nanogap.

To confirm the frequency upconversion mechanism, the \% SERS changes are also extracted for the $400-500 \mathrm{~cm}^{-1}$ spectral region (yellow/grey shaded areas for Stokes/antiStokes, Fig. 3B). These low frequency vibrational modes show no discernible change within the $\pm 10 \%$ signal noise (Fig. 3F). This lack of low wavenumber signal shows that the signal is not simply thermal heating (Fig. S5), as also suggested by the sub- $\mu$ s response, but instead is a non-equilibrium response. If simple heating were involved, a trebling of antiStokes at $1080 \mathrm{~cm}^{-1}$ would give a $60 \%$ increase at $450 \mathrm{~cm}^{-1}$, which is not observed.
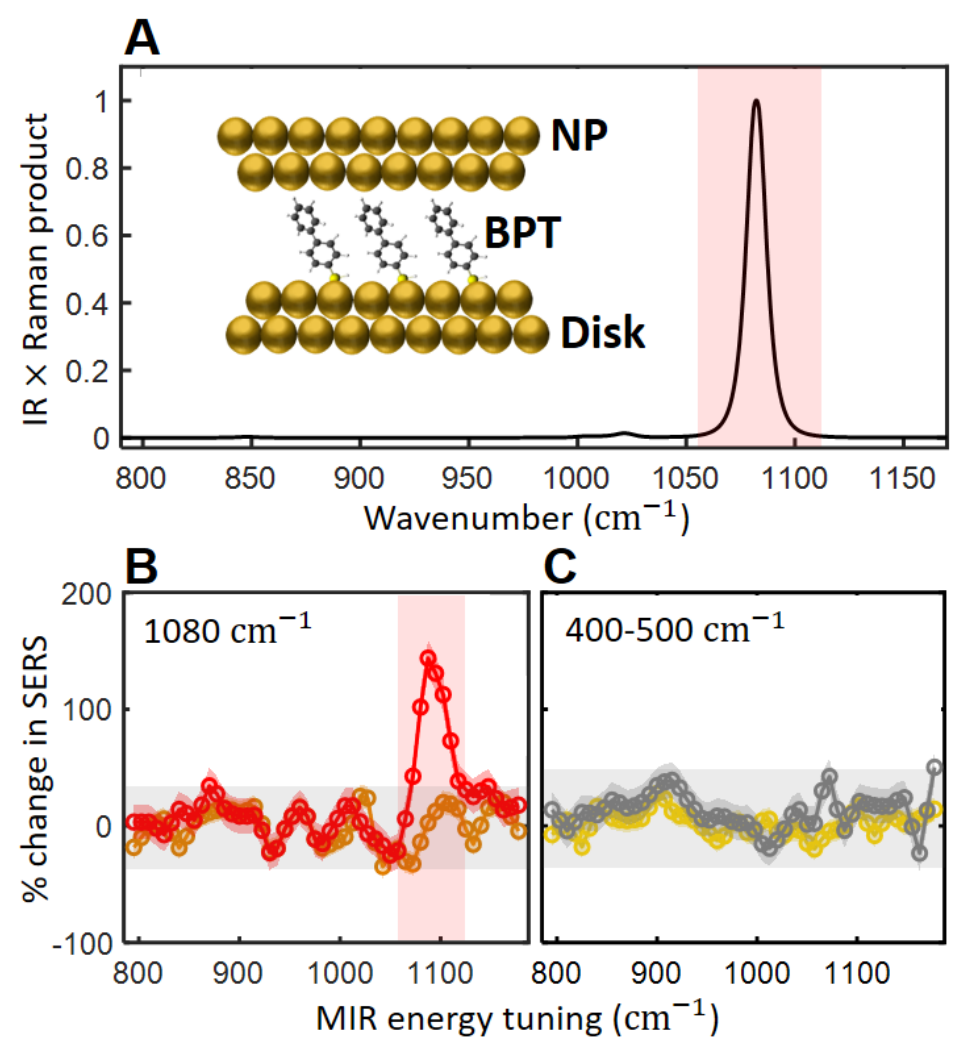

Fig. 4. MIR tuning dependence of upconversion in NPoR plasmonic construct. (A) Calculated product of molecular infrared absorption and Raman cross-section for BPT in plasmonic nanogaps (inset). (B- 
C) Percentage change in SERS from illuminated NPoR vs MIR frequency. Orange (yellow) and red (grey) correspond to SERS change of (B) $1080 \mathrm{~cm}^{-1}$ and (C) $400-500 \mathrm{~cm}^{-1}$ Stokes and antiStokes peaks.

To understand the frequency-selective dependence, we calculate the product of infrared absorption and Raman intensity of BPT, averaged over all orientations for each normal mode (Fig. 4A, SI section S1) (22). This clearly shows that the optimum overlap of optical and vibrational modes is at $1080 \mathrm{~cm}^{-1}$, and that the dipoles are all well-aligned with the vertical $E$ field in the nanogap at both visible and MIR wavelengths. To confirm this, we tune the $\mathrm{QCL}$ from $795-1170 \mathrm{~cm}^{-1}$ in $15 \mathrm{~cm}^{-1}$ steps, ensuring a constant $5 \mu \mathrm{W} / \mu \mathrm{m}^{2}$ incident on the sample. While the NPoR device shows a resonant antiStokes increase of $140 \%$ at $1080 \mathrm{~cm}^{-1}$ it shows no increase elsewhere across the frequency scan (red, Fig. 4B), and neither does the Stokes signal (orange). No clear change in SERS intensity is also seen for the $400-500 \mathrm{~cm}^{-1}$ lines across this MIR tuning on the same NPoR (Fig. 4C). This data clearly distinguishes the direct resonant pumping of the optimum $1080 \mathrm{~cm}^{-1}$ mode.

The quantum efficiency of these devices is estimated by calibrating to the thermal scale of antiStokes emission. At room temperature ( $T=300 \mathrm{~K}$ ), with MIR powers of $P=100 \mu \mathrm{W}$ (intensity $\left.I=5 \mu \mathrm{W} / \mu \mathrm{m}^{2}\right)$ at $1080 \mathrm{~cm}^{-1}(h \nu=0.13 \mathrm{eV})$, and assuming decay times from the first vibrational state of $\tau=1$ ps $(20,23)$, using the measured antiStokes increase of $\Delta \zeta=100 \%$ (see Fig. 3C) gives the fraction of MIR photons arriving that produce an observed vibration excitation as

$$
\eta=\Delta \zeta \exp \left\{-\frac{h v}{k_{B} T}\right\}\left[\left(\frac{P}{h v}\right) \tau\right]^{-1}
$$

corresponding to photon quantum efficiency $\eta \sim 2 \times 10^{-6}$ in this first generation of devices. The induced occupation of the first vibrational level is estimated to be $\Delta \zeta \exp \left\{-h v / k_{B} T\right\} \sim 1 \%$. This should be compared with the theoretical estimate (17),

$$
\eta=\eta_{\mathrm{IR}} \frac{g^{2} \tau}{\kappa_{\mathrm{IR}}} \sim 1 \times 10^{-6}
$$

using the measured MIR linewidth (Fig. 1C) to get the antenna loss rate $\kappa_{\mathrm{IR}} \sim 2.7 \mathrm{THz}$, an antenna efficiency $\eta_{\mathrm{IR}} \sim 0.5$ and an optomechanical coupling $g \sim 2 \mathrm{GHz}$ for BPT molecules in the nanocavity gap $(20,21)$. This assumes that the optical cross-section of the dualwavelength antenna matches the incident MIR focus. The main inefficiency is in the fraction of MIR photons giving significant field inside the NPoR gap to vibrationally excite a molecule. Improving the $Q$ factor of the antenna, for example using hybridization with photonic cavities, is needed for further enhancements (24).

We also show it is possible to fabricate these integrated NPoR detectors using SiN waveguides on standard 4" Si wafers (25), by cheap and scalable combination of top-down and bottomup lithographies (Fig. S3). Prospects for wideband operation are promising, with lower frequency antiStokes emission already observed at $250 \mathrm{~cm}^{-1}$ (see Fig. $3 \mathrm{~B}, \lambda=40 \mu \mathrm{m}$ or $7.5 \mathrm{THz}$ ). Utilising alternative molecules embedded in NPoRs, SERS lines observed $\sim 160 \mathrm{~cm}^{-1}$ access targets for astronomical detectors $(\mathrm{OH}$ line at 4.7THz and lower). Although the lifetime of such devices is not yet fully characterised, it already exceeds 1 week, depending on suitable encapsulation to exclude oxygen. The rapid relaxation of non-resonant molecules in the 
virtual Raman process is encouraging for engineering robust performance. We emphasise further increases in sensitivity can come from exploiting single-atom picocavities, which deliver hundred-fold larger SERS signals from the enhanced light localisation around single $\mathrm{Au}$ adatoms $(26,27)$, with simple estimates based on eqn (2) using the measured $g \sim 5 \mathrm{THz}(20$, 21) giving near unity upconversion efficiencies. This makes current efforts to stabilise picocavities significant, as well as optimising the overlap of MIR light in the same volume.

\section{REFERENCES AND NOTES}

1. M. J. Elrod, Greenhouse warming potentials from the infrared spectroscopy of atmospheric gases. J. Chem. Educ. 76, 1702 (1999).

2. A. Kuze, H. Suto, M. Nakajima, T. Hamazaki, Thermal and near infrared sensor for carbon observation Fourier-transform spectrometer on the Greenhouse Gases Observing Satellite for greenhouse gases monitoring. Appl. Opt 48, 6716-6733 (2009).

3. R. S. Allison, J. M. Johnston, G. Craig, S. Jennings, Airborne optical and thermal remote sensing for wildfire detection and monitoring. Sensors 16, 1310 (2016).

4. W. R. Bandeen, R. Hanel, J. Licht, R. Stampfl, W. Stroud, Infrared and reflected solar radiation measurements from the TIROS II meteorological satellite. J. Geophys. Res. 66, 3169-3185 (1961).

5. R. Hanel et al., The Nimbus 4 infrared spectroscopy experiment: 1. Calibrated thermal emission spectra. J. Geophys. Res. 77, 2629-2641 (1972).

6. A. Rogalski, Infrared detectors: an overview. Infrared Phys. Technol. 43, 187-210 (2002).

7. J. S. Dam, P. Tidemand-Lichtenberg, C. Pedersen, Room-temperature mid-infrared singlephoton spectral imaging. Nat. Photonics 6, 788-793 (2012).

8. I. Kviatkovsky, H. M. Chrzanowski, E. G. Avery, H. Bartolomaeus, S. Ramelow, Microscopy with undetected photons in the mid-infrared. Sci. Adv. 6, eabd0264 (2020).

9. S. Junaid et al., Video-rate, mid-infrared hyperspectral upconversion imaging. Optica 6, 702708 (2019).

10. M. Forsch et al., Microwave-to-optics conversion using a mechanical oscillator in its quantum ground state. Nat. Physics 16, 69-74 (2020).

11. J. Bochmann, A. Vainsencher, D. D. Awschalom, A. N. Cleland, Nanomechanical coupling between microwave and optical photons. Nat. Physics 9, 712-716 (2013).

12. W. Jiang et al., Efficient bidirectional piezo-optomechanical transduction between microwave and optical frequency. Nat. Communications 11, 1-7 (2020).

13. L. Shao et al., Microwave-to-optical conversion using lithium niobate thin-film acoustic resonators. Optica 6, 1498-1505 (2019).

14. F. Yesilkoy et al., Ultrasensitive hyperspectral imaging and biodetection enabled by dielectric metasurfaces. Nat. Photonics 13, 390-396 (2019).

15. A. Tittl et al., Imaging-based molecular barcoding with pixelated dielectric metasurfaces. Science 360, 1105-1109 (2018).

16. B. Schwarz et al., Monolithically integrated mid-infrared lab-on-a-chip using plasmonics and quantum cascade structures. Nat. Communications 5, 1-7 (2014).

17. P. Roelli, D. Martin-Cano, T. J. Kippenberg, C. Galland, Molecular Platform for Frequency Upconversion at the Single-Photon Level. Phys. Rev. X 10, 031057 (2020).

18. J. J. Baumberg, J. Aizpurua, M. H. Mikkelsen, D. R. Smith, Extreme nanophotonics from ultrathin metallic gaps. Nat. Materials 18, 668-678 (2019).

19. A. Xomalis et al., Interfering Plasmons in Coupled Nanoresonators to Boost Light Localization and SERS. Nano Lett. 21, 2512-2518 (2021). 
20. A. Lombardi et al., Pulsed Molecular Optomechanics in Plasmonic Nanocavities: From Nonlinear Vibrational Instabilities to Bond-Breaking. Phys. Rev. X 8, 011016 (2018).

21. F. Benz et al., Single-molecule optomechanics in "picocavities". Science 354, 726-729 (2016).

22. Z. Koczor-Benda et al., Molecular Screening for Terahertz Detection with Machine Learningbased Methods. In Review, (2021).

23. S. Yampolsky et al., Seeing a single molecule vibrate through time-resolved coherent antiStokes Raman scattering. Nat. Photonics 8, 650-656 (2014).

24. A. I. Barreda et al., Hybrid Photonic-Plasmonic Cavities based on the Nanoparticle-on-a-Mirror Configuration. arXiv preprint: 2106.01931 (2021), (2021).

25. J. Losada et al., SERS Detection via Individual Bowtie Nanoantennas Integrated in $\mathrm{Si}_{3} \mathrm{~N}_{4}$ Waveguides. IEEE J. Sel. Top. Quantum Electron. 25, 1-6 (2019).

26. T. Wu, W. Yan, P. Lalanne, Bright plasmons with cubic nanometer mode volumes through mode hybridization. ACS Photonics 8, 307-314 (2021).

27. T. Wu, M. Gurioli, P. Lalanne, Nanoscale Light Confinement: the Q's and V's. ACS Photonics, (2021).

\section{ACKNOWLEDGMENTS}

Funding: We acknowledge support from European Research Council (ERC) under Horizon 2020 research and innovation programme THOR (829067), POSEIDON (861950) and PICOFORCE (883703). We acknowledge funding from the EPSRC (Cambridge NanoDTC EP/L015978/1, EP/L027151/1, EP/S022953/1, EP/P029426/1, and EP/R020965/1). X.Z. acknowledges support from KU Leuven Internal Funds C14/19/083, IDN/20/014, KA/20/019 and FWO G090017N. R.C. acknowledges support from Trinity College, University of Cambridge. Z.K.B. and E.R. acknowledge funding from EPSRC (EP/R013012/1, EP/L027151/1) and ERC project 757850 BioNet. We are grateful to the UK Materials and Molecular Modelling Hub for computational resources, which is partially funded by EPSRC (EP/P020194/1). Author contributions: AX fabricated the devices, performed the experiments and data analysis. ZX obtained analytical electromagnetic calculations. RC and AX did full-wave simulations. SKB performed DFT calculations. EM and AX performed SERS of NPoR on Si chips. ER, AM, JJB designed and supervised the work. All authors discussed the results, provided feedback and contributed to the writing of the manuscript. Competing interests: The authors declare no competing financial interest. Data and materials availability: All data needed to evaluate the conclusions in the study are present in the main text or the supplementary materials. Source data can be found at:

\section{SUPPLEMENTARY MATERIALS}

Publications website at DOI:

Materials and Methods

Supplementary Text

Figs. S1 to S7 